Journal of Correctional Health Care 2019, Vol. 25(I) 4-14

\section{Best Practices for Pregnant Incarcerated Women With Opioid Use Disorder}

Article reuse guidelines: sagepub.com/journals-permissions DOI: 10.1 I77/10783458|8819855 journals.sagepub.com/home/jcx

(SAGE

\author{
Mary Peeler, BSPH', Kevin Fiscella, MD, $\mathrm{MPH}^{2}$, \\ Mishka Terplan, MD, MPH ${ }^{3}$, \\ and Carolyn Sufrin, MD, $\mathbf{P h D}^{4}$
}

\begin{abstract}
Pregnant women represent a unique population for correctional facilities to care for. The incarcerated pregnant population is at an increased risk of concurrent opioid use disorder (OUD) that requires specialized care. The evidence-based best practice and standard of care for pregnant women with OUD is medication-assisted treatment (MAT) with methadone or buprenorphine pharmacotherapy. Correctional facilities that house women must be prepared to provide this care in a timely manner upon intake in order to address the serious medical needs of the pregnant woman and her fetus. Providing MAT in the incarceration setting has distinctive logistics that must be considered. This article recommends strategies to optimize the care of pregnant incarcerated women with OUD, emphasizing the importance of appropriate counseling and treatment with opioid agonist pharmacotherapy.
\end{abstract}

\title{
Keywords
}

incarcerated women, pregnancy, opioid use disorder, medication-assisted treatment, correctional health care

\section{Background}

Incarcerated women (those held in federal or state prisons, jails, or juvenile detention facilities) make up one of the most vulnerable segments of society, with higher rates of mental illness, drug addiction, and histories of trauma than the general population or the incarcerated men (Sufrin, Kolbi-

\footnotetext{
I The Johns Hopkins University School of Medicine, Baltimore, MD, USA

${ }^{2}$ Family Medicine Research Programs, University of Rochester Medical Center, Rochester, NY, USA

${ }^{3}$ Department of Obstetrics and Gynecology and Department of Psychiatry, Virginia Commonwealth University, Richmond, VA, USA

${ }^{4}$ Department of Gynecology and Obstetrics, The Johns Hopkins University School of Medicine, Baltimore, MD, USA

Corresponding Author:

Carolyn Sufrin, MD, PhD, Department of Gynecology and Obstetrics, Johns Hopkins Bayview Medical Center, 4940 Eastern Ave., Room AI2I, Baltimore, MD 21224, USA.

Email: csufrin@jhmi.edu
} 
Molinas, \& Roth, 2015). At year-end 2016, nearly 214,000 women were behind bars in the United States (Carson, 2018; Zeng, 2018), representing approximately $30 \%$ of the world's total incarcerated population of women (Walmsley, 2015). The number of incarcerated women in the United States has risen by more than $700 \%$ between 1980 and 2014, outpacing the increase in men by more than $50 \%$ (Sentencing Project, 2015). The rate of female incarceration continues to rise even as that for males declines (Carson, 2018; Zeng, 2018). Women of color are disproportionately affected: Black women are incarcerated at a rate 2.3 times that of White women, and Hispanic women are incarcerated at a rate 1.5 times that of White women (Carson, 2018).

Three quarters of imprisoned women are 18 to 44 years old, prime childbearing years (Carson, 2018). Thus, many women enter prison or jail already pregnant. Systematic data on the number of incarcerated women who are pregnant are limited, but surveys from Department of Justice Bureau of Justice Statistics surveys found that $5 \%$ of women in jail, $4 \%$ in state prison, and $3 \%$ in federal prison report being pregnant upon intake (Maruschak, 2006, 2008).

One factor that complicates pregnancy - especially among incarcerated women - is drug use. Incarcerated women in general report much higher rates of substance use than the general population, with up to $90 \%$ reporting a "drug problem" (Staton, Leukefeld, \& Webster, 2003). A study of drug use and dependence among people incarcerated in state prisons and jails found that women were more likely than men to have used drugs in the month before the incarceration (Bronson, Stroop, Zimmer, \& Berzofsky, 2017). The vast majority of incarcerated women are imprisoned for nonviolent crimes, with drug offenses (such as possession or trafficking) the most serious offense for $25.1 \%$ of women in custody at the state level and $59 \%$ of women in federal prison (Carson \& Anderson, 2015).

Correctional facilities that house pregnant women therefore must be prepared to address the needs of pregnant women with concurrent drug use, particularly opioid use disorder (OUD), which presents a serious threat to the health of incarcerated pregnant women. Medication-assisted treatment (MAT), described in later sections, is the recommended treatment for pregnant women with OUD (American College of Obstetrics and Gynecology [ACOG], 2017; American Society of Addiction Medicine [ASAM], 2015; Substance Abuse and Mental Health Services Administration [SAMHSA], 2012; World Health Organization [WHO], 2014). There are many complexities to providing this recommended care in an incarceration setting. In this article, we highlight these key issues and recommend strategies that correctional facility health administrators and providers can use to optimize their care of pregnant incarcerated women with OUD, emphasizing the importance of appropriate counseling and treatment with opioid agonist pharmacotherapy.

\section{OUD in Correctional Settings}

Opioids are a growing drug of misuse in the United States. Opioid use that involves an inability to control or limit use, increased tolerance, physical dependence including withdrawal symptoms upon cessation, and continued use in the face of negative consequences falls under the maladaptive pattern of use known as OUD (SAMHSA, 2015).

In the United States, an estimated 1.9 million people have a substance use disorder involving prescription opioid pain relievers and 586,000 people have heroin use disorder (Center for Behavioral Health Statistics and Quality, 2015), up from an estimated 166,000 in 2004 (SAMHSA, 2005). In 2015, there were 33,091 overdose deaths in the United States related to prescription opioid analgesics or heroin, more than quadruple the number in 1999 (Rudd, Seth, David, \& Scholl, 2016). The so-called opioid epidemic has grown faster among women than the general U.S. population (Office on Women's Health, 2016) as has the overdose death rate (increasing $400 \%$ for women compared with 265\% for men from 1999 to 2010; Centers for Disease Control and Prevention [CDC], 2018). This gender disparity may be due to the fact that women are more likely to suffer 
from chronic pain, are more likely to be prescribed opioids for analgesia (both at higher doses and for longer duration), and may proceed from use to misuse and addiction more quickly than men (CDC, 2018).

Because illicit substance use or possession is grounds for imprisonment, there is a large overlap between drug use and incarceration. Up to one third of all people who use heroin in the United States are involved in the criminal justice system (Rich, Wakeman, \& Dickman, 2011). About one in four females in state prison or jail reported regular use of heroin/opiates (Bronson et al., 2017). The high rates of opioid use among female arrestees make it likely that some may be carrying an opioidexposed pregnancy.

OUD is a complex condition with neurological as well as social and environmental components (Volkow, Koob, \& McLellan, 2016). Thus, approaches to treatment must also be multifactorial. The evidence-based best practice for the treatment of OUD is MAT (Kampman \& Jarvis, 2015; Schuckit, 2016). MAT combines behavioral therapy and medication to treat OUD. It requires long-term support and counseling in addition to medication.

MAT, particularly methadone and buprenorphine, brings benefits and mitigates risks for people with OUD. Carefully monitored MAT can reduce if not eliminate intravenous (IV) drug use and its attendant risks including infectious disease acquisition and transmission, prevent withdrawal symptoms, reduce cravings, and improve quality of life for people with OUD (Kampman \& Jarvis, 2015; SAMHSA, 2014). Randomized controlled trial data support the continuation of medication among newly incarcerated people who were enrolled in a methadone maintenance program in the community (Rich et al., 2015). Continuing methadone treatment in correctional settings has been associated with decreased levels of drug use, criminal activity, and recidivism upon release (Chandler, Fletcher, \& Volkow, 2009). Discontinuation of opioids without MAT is associated with a high risk of relapse (Day \& Strang, 2011). Relapse followed by overdose is the leading cause of the dramatic spike in deaths in the weeks following prison release (Binswanger, Blatchford, Mueller, \& Stern, 2013), with much greater risk for women (Groot et al., 2016). Despite these benefits, most correctional facilities do not offer MAT, even to individuals who enter the system on methadone pharmacotherapy (Mitchell et al., 2009).

\section{OUD in Pregnancy}

Opioid use by pregnant women has paralleled increases in the general population. Maternal opioid use during pregnancy quadrupled from 1999 to 2014, from 1.5 per 1,000 delivery hospitalizations to 6.5 (Haight et al., 2018). Pregnant women with OUD have unique health needs. In addition to the effects of the opioids on the pregnant woman herself, substance use during pregnancy is associated with higher rates of pregnancy complications including fetal growth restriction, placental abruption, preterm labor, or fetal death (ACOG, 2017). These effects are directly due to the substance itself and to the associated behaviors such as smoking, poor nutrition, a lack of prenatal care, and needle sharing (ACOG, 2017). Intravenous and intranasal drug administration is a significant risk factor for infections that can endanger both the pregnant woman and the fetus, including hepatitis $\mathrm{B}$ and $\mathrm{C}$, HIV, bacterial endocarditis, and cellulitis (SAMHSA, 2012).

Due to these risks, screening for and treatment of OUD during pregnancy are of paramount importance. Opioid-dependent patients who abruptly stop using opioids will suffer withdrawal symptoms such as severe nausea or vomiting, muscle aches, diarrhea, fever, dehydration, and insomnia, as well as strong cravings that can occur when people first enter jail or prison (SAMHSA, 2011). Data from two systematic reviews of detoxification in pregnancy reported no alteration in the rate of preterm birth or fetal loss with detoxification (Terplan et al., 2018; Wang et al., 2019). However, detoxification completion rates were low and there was no evidence that detoxification prevented NAS. Most importantly, relapse rates were extremely high, which can lead to overdose 
and other known risks of illicit opioid use. Even medication-assisted withdrawal (also known as medication-assisted detoxification) in pregnancy increases the risk of maternal relapse without improvement in newborn health (Jones, Terplan, \& Meyer, 2017; Zedler et al., 2016). The use of opioids such as oxycodone or codeine to quell withdrawal symptoms in pregnancy is likewise not appropriate. Thus, detoxification is not recommended in pregnancy and MAT remains the standard of care.

MAT can prevent these withdrawal symptoms and accompanying complications. MAT in pregnancy is associated with improved adherence to addiction treatment, prenatal care, in-hospital delivery, decreased risk to HIV and hepatitis, and reduced risk of relapse and overdose (Jones, O’Grady, Malfi, \& Tuten, 2008; Krans, Cochran, \& Bogen, 2015). Therefore, continued MAT with methadone or buprenorphine throughout the duration of the pregnancy is considered primary best treatment for OUD by ASAM (Kampman \& Jarvis, 2015), ACOG (2017), the National Commission on Correctional Health Care (NCCHC, 2016), and WHO (2014).

The Estelle v. Gamble (1976) Supreme Court case established that correctional facilities are constitutionally mandated to tend to incarcerated individuals' "serious medical needs;" this extends to the care of pregnant women. Given that MAT is evidence-based best practice for treating OUD in pregnancy, it should also be so in correctional settings. Indeed, the standards from NCCHC (2016) advise that pregnant women with OUD should not undergo detoxification and should be offered MAT. Similarly, national guidelines for opioid treatment in corrections and a National Governors Association report that recommended access to MAT for all incarcerated individuals in correctional facilities indicates a shift toward MAT becoming a standard of care in corrections (Kampman \& Jarvis, 2015; Murphy et al., 2016). Accordingly, pregnant women who were on pharmacotherapy during pregnancy should not be detoxified after their pregnancies end, and should continue methadone or buprenorphine.

Despite these recommendations, many pregnant incarcerated women do not have access to MAT. Half of the 28 prison systems that have MAT do so only for pregnant women but $45 \%$ of prison systems still do not provide MAT at all (Nunn et al., 2009). Likewise, a survey of policies at 53 jails across the country found that nearly half of them required pregnant women to withdraw (Kelsey, Medel, Mullins, Dallaire, \& Forestell, 2017). Only one third permitted women to continue methadone if they had been prescribed it before incarceration and even fewer $(22 \%)$ initiated methadone in jail (Kelsey et al., 2017).

\section{MAT Approaches and Concerns}

Methadone, buprenorphine, and naltrexone are the three MAT medications approved by the U.S. Food and Drug Administration (FDA). Both methadone and buprenorphine have been studied for their safety and effectiveness in pregnant populations, whereas naltrexone is not recommended in pregnancy.

\section{Methadone}

Methadone has been used for over 40 years to treat OUD, including in pregnant patients. It has been well studied in pregnancy and is not associated with teratogenic effects on the fetus (SAMHSA, 2012). It is an opioid receptor agonist, lessening symptoms of withdrawal and reducing effects of other opioids such as heroin, morphine, or oxycodone (SAMHSA, 2018). Its effectiveness is supported by meta-analyses of randomized trials (Mattick, Breen, Kimber, \& Davoli, 2009).

Methadone can only be prescribed for the outpatient treatment of OUD through an opioid treatment program (OTP). OTPs must be certified by SAMHSA and registered with the Drug Enforcement Administration (DEA). Highlighting the importance of concordant 
nonpharmacological therapies, the federal government requires that OTPs provide counseling services including vocational, educational, and medication counseling (SAMHSA, 2018).

\section{Buprenorphine}

The FDA approved buprenorphine for the treatment of OUD in 2002 (SAMHSA, 2018). Buprenorphine is a partial opioid agonist, meaning that it binds to opioid receptors producing a weaker effect than full agonists like methadone. It also has a "ceiling effect," meaning that the opioid effects increase with dosage to a certain threshold at which a larger dose does not produce a larger response, reducing the potential for misuse and death from overdose (SAMHSA, 2018). Like methadone, buprenorphine's effectiveness is also supported by meta-analyses, though some studies suggest that patients may be less likely to remain on buprenorphine long term than on methadone (Mattick et al., 2009).

Buprenorphine can also be used during pregnancy; research has found it to be "well accepted by mothers and infants ... and useful to treat pregnant women who were opioid addicted" (SAMHSA, 2012, p. 221). Data to support equivalence of buprenorphine compared to methadone include several cohorts, a randomized trial, and a meta-analysis, which found that buprenorphine was associated with a lower risk of preterm birth and greater birth weight compared to methadone with no associated increased harms (Zedler et al., 2016). Some women are unable or unwilling to take methadone or are already on stable maintenance with buprenorphine. Buprenorphine can and should be used in pregnant patients under these circumstances (SAMHSA, 2012).

Buprenorphine is typically formulated in combination with naloxone, an opioid receptor antagonist, and sold under the brand name Suboxone. Because there is little research on the effects of naloxone on fetal development, buprenorphine monoproduct (Subutex) is generally preferred in pregnant women (SAMHSA, 2012).

Buprenorphine is more accessible than methadone for prescribing; it can be prescribed through an OTP, like methadone, or by community physicians who have received an 8-hour training and have a special DEA waiver. In 2016, Congress expanded prescribing authority to nurse practitioners and physician assistants who complete required certification (ASAM, 2016). Buprenorphine is more easily accessible for patients because they do not have to be enrolled in an OTP and can simply be under the care of a trained primary care physician. However, ensuring continuity in treatment with community prescribers following the woman's release can pose a challenge.

\section{Naltrexone}

Naltrexone is a full opioid antagonist that is available in oral or injectable depot formulations and is approved by the FDA for the treatment of OUD. While its efficacy has been shown (Lee et al., 2016; Schuckit, 2016), its effectiveness is limited by nonadherence (Fiscella, 2016; Minozzi et al., 2011). It is not recommended in pregnancy due to the need to withdraw from all opioids before it can be initiated, potential morbidity and mortality risks to the woman and her fetus if she drops out of naltrexone treatment, and unknown direct risks to the fetus (Waal, 2013).

\section{Neonatal Abstinence Syndrome}

Any continued opioid exposure during pregnancy - whether prescription analgesic, heroin, or MAT - poses a risk of withdrawal and neonatal abstinence syndrome (NAS) after delivery (SAMHSA, 2012). NAS rates have nearly quadrupled between 2004 and 2013 (Tolia et al., 2015). Symptoms of withdrawal may appear from immediately after birth to 2 weeks of life and may include difficulty feeding, tremors, and sleep disturbances (SAMHSA, 2012). NAS is highly 
varied and depends on many contributing factors outside of the mother's MAT usage and is not dependent on the mother's methadone dosage (Mitchell et al., 2009). A double-blind, randomized controlled study found buprenorphine to be associated with lower rates and lower severity of NAS than methadone when taken throughout pregnancy (Jones et al., 2010).

Infants born to mothers using opioids or MAT are observed closely in the first hours and days of life for signs of NAS. The severity of NAS can be measured with the Neonatal Abstinence Score, and symptoms can be treated through pharmacological management and gradual weaning from opioids (SAMHSA, 2012). Importantly, SAMHSA (2012) notes that "NAS can be treated satisfactorily without any severe neonatal outcomes," meaning that it is a manageable complication of MAT use in pregnancy (p. 219). Additionally, methadone and buprenorphine are minimally transmitted in breast milk, and barring other contraindications, breastfeeding should be encouraged for mothers on MAT (SAMHSA, 2012).

\section{Recommendations for MAT for Pregnant Women in Correctional Settings}

Given that MAT is the evidence-based standard of care in pregnancy, correctional facilities that house women who may have co-occurring OUD and pregnancy must be prepared to provide MAT. Successful MAT for OUD is typically a long-term process with many different interventions including support groups, cognitive behavioral therapy, or contingency management, as well as regular monitoring to support a patient receiving methadone or buprenorphine (National Institute on Drug Abuse, 2012). Correctional facilities should invest resources in multifaceted programs for their pregnant patients, as this will have benefits not only for the expectant mothers but also for their newborns.

Failing to provide this standard of care could expose providers to the possibility of medical-legal consequences. If a facility fails to provide MAT, the accepted standard of care, there should be justifiable reason and clear documentation.

However, there are some logistics unique to correctional facilities to consider, including the importance of rapid evaluation and treatment. Heroin users may begin to experience withdrawal symptoms as soon as 8 hours after their last dose (WHO, 2009). Pregnant women who are newly incarcerated should receive timely medical care so that they can be prevented from undergoing acute withdrawal, as inaction or delay in treatment will lead to withdrawal. Because of this, correctional facilities should have a plan in place for screening women for both pregnancy and OUD upon intake.

Correctional health providers need to have the appropriate arrangements to legally provide methadone and buprenorphine. Federal OTP regulations allow MAT professionals to supervise treatment in correctional facilities that lack an OTP license as long as the facility has a working relationship with a certified OTP (SAMHSA, 2018). Within these federal requirements, correctional facilities have several options for treating pregnant women with MAT.

The first option is to transport the woman to a community OTP or a hospital for daily treatment. OTPs may obtain waivers from SAMHSA for the use of take-home doses that are administered under the custody of the jail or prison in order to minimize the number of transports. This option may present a logistical challenge, given the frequency of off-site transports and the availability of slots in local OTPs.

Second, correctional facilities can partner with community OTPs for dosing of incarcerated people within the facility. In this case, the dosing is done under the license of the community OTP. This requires transport of methadone doses to the correctional facility for custody. Doses are secured, carefully tracked, and administered by correctional health care staff. Unused doses (when people are released unexpectedly) are returned to the OTP for accounting. 
Third, correctional clinicians with buprenorphine licenses can prescribe it for people in custody. This license permits the use of buprenorphine for MAT or for withdrawal (for people preferring this option). However, women who are on methadone at community OTPs should generally be continued on methadone due to the risk of precipitating withdrawal when transitioning from methadone to buprenorphine.

Fourth, the correctional health services unit can obtain a facility license to operate as an OTP. This license permits the dispensing of both methadone and buprenorphine for incarcerated individuals including those who are pregnant. It also allows for the use of these medications for tapered withdrawal if a pregnant woman declines maintenance. The accreditation process is rigorous, and only a handful of jails and prisons have obtained OTP accreditation.

Last, if the health care services unit within the correctional facility has obtained appropriate state and federal licenses to operate as a health care facility, this entitles the facility to the same exemptions as hospitals for the use of methadone or buprenorphine for maintenance or withdrawal during pregnancy or to ensure treatment of other conditions, for example, HIV or mental illness (DEA, 2000). However, most jails and prisons do not operate their health care facilities under such licenses that would permit such an exemption.

Each of these strategies requires investment of resources from the correctional facility, but the variety of options should provide an appropriate choice for each facility so that all pregnant women have access to MAT if they consent to it. Correctional facilities can partner with local providers and regulatory agencies in order to create the best systems for providing MAT for pregnant women in their facilities. Although implementing MAT involves costs for medications, partnerships, and transports if off-site, there are also potential cost savings with regard to decreased use of medications and supervising personnel for detoxification.

Although MAT is the evidence-based best practice for women with OUD, no woman should be forced into treatment. Given the nuances of autonomy in and the inherently controlling environment of correctional settings, providers must take special care to ensure that their treatment recommendation for MAT is not coercive. Improved education and training of clinicians on the right of informed refusal should enable health care providers to appropriately counsel pregnant patients with OUD on the risks to them and the developing fetus of withdrawal and relapse, and the benefits and risks of MAT use, including NAS. Correctional institutional policies should emphasize the woman's right of informed refusal. If a woman declines MAT, medically supervised withdrawal should be managed by an experienced physician, include fetal monitoring when indicated, and involve continued behavioral counseling to prevent relapse (ACOG, 2017). All possible measures should be undertaken to help prevent relapse, such as support resources common in MAT, including cognitive behavioral therapy, group counseling, or a therapeutic community (Kouyoumdjian et al., 2015).

OUD is a chronic brain disease (Volkow et al., 2016); consequently, methadone or buprenorphine maintenance usually requires long-term treatment. As individuals move in and out of custody, their treatment should be consistently maintained (Kampman \& Jarvis, 2015). Treatment with MAT should be continued throughout the pregnancy, and care should be taken to ensure continuity with a community provider if the woman is released.

\section{Summary}

Treatment of OUD in pregnant incarcerated women is an emerging and important health issue for correctional facilities. The evidence-based treatment of OUD in pregnant women is MAT with either methadone or buprenorphine and associated mental health services such as cognitive behavioral therapy and group sessions. 
Correctional facilities have a number of options that allow health care providers to ensure that pregnant women receive MAT. They can partner with a community OTP either by arranging transport for patients or by arranging dosing inside the facility. Correctional clinicians with buprenorphine licenses can prescribe it to people in custody. Correctional health services can apply for a license to operate as an OTP or as an independent health care facility with the hospital exemption for MAT provision.

Despite these options and proven benefits of MAT, many prisons and jails do not offer methadone or buprenorphine to newly incarcerated women with OUD or patients enrolled in methadone maintenance programs, leading to acute opioid withdrawal (Mitchell et al., 2009). The consequences of withdrawal are especially grave in pregnant women. Providing MAT for pregnant patients will match the community standard of care, improve the health of a vulnerable group of pregnant women, and work toward optimal health outcomes for both women and their newborns.

\section{Declaration of Conflicting Interests}

The authors disclosed no conflicts of interest with respect to the research, authorship, or publication of this article. For information about $J C H C$ 's disclosure policy, please see the Self-Study Program.

\section{Funding}

The authors disclosed receipt of the following financial support for the research, authorship, and/or publication of this article: Dr. Sufrin received funding from NICHD-K12HD085845.

\section{References}

American College of Obstetricians and Gynecologists Committee on Obstetric Practice \& American Society of Addiction Medicine. (2017). Opinion No. 711: Opioid use and opioid use disorder in pregnancy. Obstetrics and Gynecology, 130, e81-e94.

American Society of Addiction Medicine. (2015). The national practice guideline for the use of medications in the treatment of addiction involving opioid use. Retrieved from http://www.asam.org/docs/default-source/ practice-support/guidelines-and-consensus-docs/asam-national-practice-guideline-supplement.pdf

American Society of Addiction Medicine. (2016). Nurse practitioners and physician assistants prescribing buprenorphine. Retrieved from http://www.asam.org/quality-practice/practice-resources/nurse-practi tioners-and-physician-assistants-prescribing-buprenorphine

Binswanger, I. A., Blatchford, P. J., Mueller, S. R., \& Stern, M. F. (2013). Mortality after prison release: Opioid overdose and other causes of death, risk factors, and time trends from 1999 to 2009. Annals of Internal Medicine, 159, 592-600.

Bronson, J., Stroop, J., Zimmer, S., \& Berzofsky, M. (2017). Drug use, dependence, and abuse among state prisoners and jail inmates, 2007-2009 (NCJ 250546). Washington, DC: Bureau of Justice Statistics.

Carson, E. A., \& Anderson, E. (2015). Prisoners in 2015 (NCJ 250229). Washington, DC: Bureau of Justice Statistics.

Carson, E. A. (2018). Prisoners in 2016 (NCJ 251149). Washington, DC: Bureau of Justice Statistics.

Center for Behavioral Health Statistics and Quality. (2015). Behavioral health trends in the United States: Results from the 2014 National Survey on Drug Use and Health (HHS Publication No. SMA 15-4927, NSDUH Series H-50). Rockville, MD. Retrieved from https://www.samhsa.gov/data/sites/default/files/ NSDUH-FRR1-2014/NSDUH-FRR1-2014.htm

Centers for Disease Control and Prevention. (2018). Prescription painkiller overdoses: A growing epidemic, especially among women. Atlanta, GA: Author. Retrieved from http://www.cdc.gov/vitalsigns/prescription painkilleroverdoses/index.html

Chandler, R. K., Fletcher, B. W., \& Volkow, N. D. (2009). Treating drug abuse and addiction in the criminal justice system: Improving public health and safety. Journal of the American Medical Association, 301, 183-190. doi:10.1001/jama.2008.976 
Day, E., \& Strang, J. (2011). Outpatient versus inpatient opioid detoxification: A randomized controlled trial. Journal of Substance Abuse Treatment, 40, 56-66.

Drug Enforcement Administration. (2000). Narcotic treatment programs best practice guideline. Retrieved from https://www.deadiversion.usdoj.gov/pubs/manuals/narcotic/narcotic.pdf

Estelle v. Gamble 429 U.S. 97 (1976).

Fiscella, K. (2016). Guidelines and quality measures should reflect evidence for effectiveness of naltrexone. Journal of Addiction Medicine, 10, 453.

Groot, E., Kouyoumdjian, F. G., Kiefer, L., Madadi, P., Gross, J., Prevost, B., .. Persaud, N. (2016). Drug toxicity deaths after release from incarceration in Ontario, 2006-2013: Review of coroner's cases. PLoS One, 11, e0157512. doi:10.1371/journal.pone.0157512

Haight, S. C., Ko, J. Y., Tong, V. T., Bohm, M. K., \& Callaghan, W. M. (2018). Opioid use disorder documented at delivery hospitalization-United States, 1999-2014. Morbidity and Mortality Weekly Report, $67,1-5$.

Jones, H. E., Kaltenbach, K., Heil, S. H., Stine, S. M., Coyle, M. G., Arria, A. M., ...Fischer, G. (2010). Neonatal abstinence syndrome after methadone or buprenorphine exposure. New England Journal of Medicine, 363, 2320-2331. doi:10.1056/NEJMoa1005359

Jones, H. E., O’Grady, K. E., Malfi, D., \& Tuten, M. (2008). Methadone maintenance vs. methadone taper during pregnancy: Maternal and neonatal outcomes. American Journal on Addictions, 17, 372-386. doi:10. 1080/10550490802266276

Jones, H. E., Terplan, M., \& Meyer, M. (2017). Medically assisted withdrawal (detoxification): Considering the mother-infant dyad. Journal of Addition Medicine, 11, 90-92. doi:10.1097/ADM.0000000000000289

Kampman, K., \& Jarvis, M. (2015). American Society of Addiction Medicine (ASAM) national practice guideline for the use of medications in the treatment of addiction involving opioid use. Journal of Addiction Medicine, 9, 358-367.

Kelsey, C. M., Medel, N., Mullins, C., Dallaire, D., \& Forestell, C. (2017). An examination of care practices of pregnant women incarcerated in jail facilities in the United States. Maternal and Child Health Journal, 21, 1260-1266. doi:10.1007/s10995-016-2224-5

Kouyoumdjian, F. G., McIsaac, K. E., Liauw, J., Green, S., Karachiwalla, F., Siu, W., . . Hwang, S. W. (2015). A systematic review of randomized controlled trials of interventions to improve the health of persons during imprisonment and in the year after release. American Journal of Public Health, 105, e13-e33.

Krans, E. E., Cochran, G., \& Bogen, D. L. (2015). Caring for opioid-dependent pregnant women: Prenatal and postpartum care considerations. Clinical Obstetrics and Gynecology, 58, 370-379. doi:10.1097/GRF. 0000000000000098

Lee, J. D., Friedmann, P. D., Kinlock, T. W., Nunes, E. V., Boney, T. Y., Hoskinson, R. A. Jr., . . O’Brien, C. P. (2016). Extended-release naltrexone to prevent opioid relapse in criminal justice offenders. New England Journal of Medicine, 374, 1232-1242.

Maruschak, L. M. (2006). Medical problems of jail inmates (NCJ 210696). Washington, DC: Bureau of Justice Statistics.

Maruschak, L. M. (2008). Medical problems of prisoners (NCJ 221740). Washington, DC: Bureau of Justice Statistics.

Mattick, R. P., Breen, C., Kimber, J., \& Davoli, M. (2009). Methadone maintenance therapy versus no opioid replacement therapy for opioid dependence. Cochrane Database of Systematic Reviews, 2009, CD002209. doi:10.1002/14651858.CD002209.pub2

Minozzi, S., Amato, L., Vecchi, S., Davoli, M., Kirchmayer, U., \& Verster, A. (2011). Oral naltrexone maintenance treatment for opioid dependence. Cochrane Database of Systematic Reviews, 2011, CD001333. doi:10.1002/14651858.CD001333.pub4

Mitchell, S. G., Kelly, S. M., Brown, B. S., Reisinger, H. S., Peterson, J. A., Ruhf, A., . . Schwartz, R. P. (2009). Incarceration and opioid withdrawal: The experiences of methadone patients and out-of-treatment heroin users. Journal of Psychoactive Drugs, 41, 145-152. 
Murphy, K., Becker, M., Locke, J., Kelleher, C., McLeod, J., \& Isasi, F. (2016). Finding solutions to the prescription opioid and heroin crisis: A road map for states. Washington, DC: National Governors Association Center for Best Practices.

National Commission on Correctional Health Care. (2016). Substance use disorder treatment for adults and adolescents (Position statement). Chicago, IL. Retrieved from https://www.ncchc.org/filebin/Positions/Sub stance-Use-Disorder-Treatment-2016.pdf

National Institute on Drug Abuse. (2012). Principles of drug addiction treatment: A research-based guide (3rd ed., NIH Publication No. 12-4180). Retrieved from https://www.drugabuse.gov/publications/principlesdrug-addiction-treatment-research-based-guide-third-edition

Nunn, A., Zaller, N., Dickman, S., Trimbur, C., Nijhawan, A., \& Rich, J. D. (2009). Methadone and buprenorphine prescribing and referral practices in US prison systems: Results from a nationwide survey. Drug and Alcohol Dependence, 105, 83-88.

Office on Women's Health. (2016). White paper: Opioid use, misuse, and overdose in women. Washington, DC: U.S. Department of Health and Human Services.

Rich, J. D., McKenzie, M., Larney, S., Wong, J. B., Tran, L., Clarke, J., ...Zaller, N. (2015). Methadone continuation versus forced withdrawal on incarceration in a combined US prison and jail: A randomised, open-label trial. Lancet, 386, 350-359.

Rich, J. D., Wakeman, S. E., \& Dickman, S. L. (2011). Medicine and the epidemic of incarceration in the United States. New England Journal of Medicine, 364, 2081-2083.

Rudd, R. A., Seth, P., David, F., \& Scholl, L. (2016). Increases in drug and opioid-involved overdose deathsUnited States, 2010-2015. Morbidity and Mortality Weekly Report, 65, 1445-1452. doi:10.15585/mmwr. mm655051e1

Schuckit, M. A. (2016). Treatment of opioid-use disorders. New England Journal of Medicine, 375, 357-368.

The Sentencing Project. (2015). Incarcerated women and girls (Fact sheet). Washington, DC: Retrieved from http://www.sentencingproject.org/wp-content/uploads/2016/02/Incarcerated-Women-and-Girls.pdf

Staton, M., Leukefeld, C., \& Webster, J. M. (2003). Substance use, health, and mental health: Problems and service utilization among incarcerated women. International Journal of Offender Therapy and Comparative Criminology, 47, 224-239.

Substance Abuse and Mental Health Services Administration. (2005). Results from the 2004 National Survey on Drug Use and Health: National findings (HHS Publication No. SMA 05-4062). Rockville, MD.

Substance Abuse and Mental Health Services Administration. (2011). Medication-assisted treatment for opioid addiction: Facts for families and friends (HHS Publication No. SMA 09-4443). Rockville, MD. Retrieved from https://www.ct.gov/dmhas/lib/dmhas/publications/MAT-InfoFamilyFriends.pdf

Substance Abuse and Mental Health Services Administration. (2012). Medication-assisted treatment for opioid addiction in opioid treatment programs (Treatment Improvement Protocol [TIP] Series 43, HHS Publication No. SMA 12-4214). Rockville, MD.

Substance Abuse and Mental Health Services Administration. (2014). Methadone treatment for pregnant women (HHS Publication No. SMA 14-4124). Rockville, MD.

Substance Abuse and Mental Health Services Administration. (2015). Substance use disorders. Retrieved from https://www.samhsa.gov/disorders/substance-use

Substance Abuse and Mental Health Services Administration. (2018). Treatments for substance use disorders. Retrieved from https://www.samhsa.gov/treatment/substance-use-disorders\#opioid

Sufrin, C., Kolbi-Molinas, A., \& Roth, R. (2015). Reproductive justice, health disparities and incarcerated women in the United States. Perspectives on Sexual and Reproductive Health, 47, 213-219.

Terplan, M., Laird, H. J., Hand, D. J., Wright, T. E., Premkumar, A., Martin, C. E., ... Krans, E. E. (2018) Opioid detoxification during pregnancy: A systematic review. Obstetrics and Gynecology, 131, 803-814.

Tolia, V. N., Patrick, S. W., Bennett, M. M., Murthy, K., Sousa, J., Smith, P. B., ... Spitzer, A. R. (2015). Increasing incidence of the neonatal abstinence syndrome in U.S. neonatal ICUs. New England Journal of Medicine, 372, 2118-2126. 
Volkow, N. D., Koob, G. F., \& McLellan, A. T. (2016). Neurobiologic advances from the brain disease model of addiction. New England Journal of Medicine, 374, 363-371.

Waal, H. (2013). Is sustained release naltrexone an option for heroin-dependent pregnant women? Addiction, $108,252-253$

Walmsley, R. (2015). World female imprisonment list: Women and girls in penal institutions, including pretrial detainees/remand prisoners (3rd ed.). London, England: World Prison Brief, Institute for Criminal Policy Research. Retrieved from http://www.prisonstudies.org/sites/default/files/resources/downloads/ world_female_imprisonment_list_third_edition_0.pdf

Wang, M. J., Kuper, S. G., Sims, B., Paddock, C. S., Dantlzer, J., Muir, S., \& Harper, L. M. (2018). Opioid detoxification in pregnancy: Systematic review and meta-analysis of perinatal outcomes. American Journal of Perinatology, epub ahead of print.

World Health Organization. (2009). Clinical guidelines for withdrawal management and treatment of drug dependence in closed settings. Geneva, Switzerland: Author. Retrieved from http://www.wpro.who.int/ publications/docs/ClinicalGuidelines_forweb.pdf

World Health Organization. (2014). Guidelines for the identification and management of substance use and substance use disorders in pregnancy. Geneva, Switzerland: Author. Retrieved from http://apps.who.int/iris/ bitstream/handle/10665/107130/9789241548731_eng.pdf;jsessionid=B26585112A0F72C7C1F52 E427A6BBE50? sequence $=1$

Zedler, B. K., Mann, A. L., Kim, M. M., Amick, H. R., Joyce, A. R., Murrelle, E. L., \& Jones, H. E. (2016). Buprenorphine compared with methadone to treat pregnant women with opioid use disorder: A systematic review and meta-analysis of safety in the mother, fetus and child. Addiction, 111, 2115-2128.

Zeng, Z. (2018). Jail inmates in 2016. NCJ 251210. Washington, DC: Bureau of Justice Statistics. 